\title{
Atrial fibrillation, end-stage renal disease and hemorrhagic pleural-pericarditis
}

\author{
Raluca-Mihaela Leonte ${ }^{*}, 1$, Laurențiu Vladimir Lucaci ${ }^{1,3}$, Cristiana Elena Vlad ${ }^{2,3}$, \\ Andreea Florea ${ }^{3}$, Laura Florea ${ }^{2,3}$
}

1Department of Cardiology, "Prof. Dr. George I.M. Georgescu" Institute of Cardiovascular Diseases, Iasi, Romania, ${ }^{2}$ Department of Internal Medicine, "Dr. C.I. Parhon”, Iasi, Romania, “"Grigore T. Popa” University of Medicine and Pharmacy, Iasi, Romania

\begin{abstract}
Pericarditis is the most common pericardial disease found in clinical practice, with an incidence of acute pericarditis reported in 27.7 cases per 100,000 subjects per year. Hemodialysis in end stage renal disease (ESRD) is associated with frequent cardiovascular modifications, mostly because of the highly fluctuating levels of potassium, magnesium, ionized calcium, sodium and volume status. The risk of arrhythmias is increased and chronic atrial fibrillation (AF) can be found among approximately $14 \%$ of patients. The renal disease combined with arrhythmias increases the risk of systemic thromboembolism but also of bleeding events. Here we present the case of a male patient, with ESRD, recently diagnosed with intradialytic paroxysmal AF for which oral anticoagulation therapy is initiated, but it's early complicated with hemorrhagic pleural-pericarditis.
\end{abstract}

Keywords: hemorrhagic pleural-pericarditis, atrial fibrillation, ESRD, hemodialysis

\section{Introduction}

Pericarditis is the most common pericardial disease encountered in clinical practice. The incidence of acute pericarditis was reported in 27.7 cases per 100,000 subjects per year. Hemorrhagic fluid usually indicates neoplastic (commonly found in the US) and tuberculous (in developing countries) etiology. It can be found even in patients with acute articular rheumatism, cardiac trauma or myocardial infarction, but it is not as common in patients with chronic kidney disease (CKD) in pre-dialysis or dialysis. [1]

Received: November 2019; Accepted after review: December 2019; Published: December 2019.

${ }^{*}$ Corresponding author: Raluca-Mihaela Leonte, Department of Cardiology, "Prof. Dr. George I.M. Georgescu" Institute of Cardiovascular Diseases, 50 Carol I Bvd., lasi, Romania

Email: ralucaleonte@ymail.com
Pleurisy has a prevalence of 320 cases per 100,000 inhabitants in industrialized countries. The etiology of hemorrhagic pleurisy can be neoplastic, tuberculous, pulmonary embolism, collagenosis, ESRD or hemodialysis (HD). [2]

Hemorrhagic pleural-pericarditis consists of a concomitant fluid accumulation in the two cavities, pleural and pericardial. Depending on the amount of fluid and how long it takes for the fluid to accumulate, the symptoms and clinical signs may be mild respiratory distress and chest pain, or a noisy clinical picture in the case of cardiac tamponade (dyspnea, cough, chest pain, hypotension, dizziness, lipothymia, paradoxical pulse). [3]

The diagnosis is mainly imagistic: chest radiography, echocardiography; for a certain diagnostic pleural puncture and pericardial puncture are performed. The puncture confirms the presence of the fluid. It may have a different macroscopic appearance (sero- 
fibrinous, citrine or sero-hemorrhagic) and it allows cytological, bacteriological and biochemical examinations of the pleural. This can be, at the same time, a therapeutic act. [4]

\section{Case report}

We present the case of a 65-year-old male patient, with ESRD, chronically dialyzed for 13 years; initially peritoneal dialyzed for 10 years, then, for the last 3 years, hemodialyzed on arterio-venous fistula, which presented a lipothymic episode, accompanied by palpitations and dyspnea. $\mathrm{He}$ was then diagnosed with newly installed atrial fibrillation (AF) that occurred intradialytic $(\mathrm{K}=3.2 \mathrm{mEq} / \mathrm{I})$ and medium quantity pericarditis. The treatment was initiated for the rhythm disorder (antiarrhythmic, beta-blocker, oral anticoagulation - antivitamin K) and for the pericardial disease (anti-inflammatory Ibuprofen). The patient has a background of cardiovascular pathology (old anterior myocardial infarction treated with revascularization of the anterior descending coronary artery for which he received antiplatelet therapy that was interrupted right after the initiation of lbuprofen).

Five days after discharge, the patient has a new lipothymic episode, associated with hypotension: systolic blood pressure (SBP) $=50 \mathrm{mmHg}$. On admission, the patient was anxious, he had regular heart beats, systolic

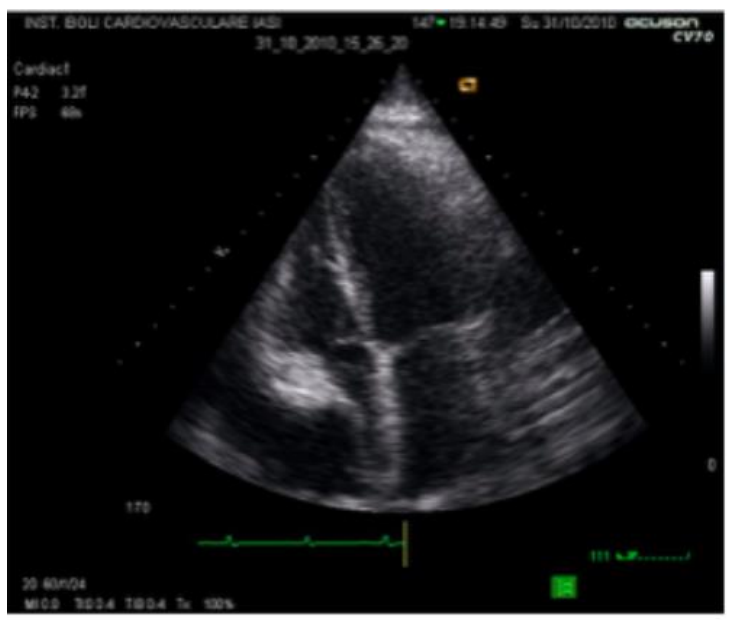

murmur in the mitral position, breath sounds were soft on the inferior half of the left hemithorax, $\mathrm{SaO}_{2}$ (arterial oxygen saturation) $=95 \%$ with oxygen therapy on the nasal cannula, he had abdominal-type respirations, congestive hepatomegaly, grade 3 turgid jugular veins, pale teguments, and no lower limbs edema. Vasopressor therapy (positive inotropic agent - Dobutamine) was initiated and the SBP increased to $100 \mathrm{mmHg}$.

Laboratory findings revealed: RBC (red blood cells) $=2.0010 * 12 / \mathrm{L}, \mathrm{Hb}$ (hemoglobin) $=$ $6.4 \mathrm{~g} / \mathrm{dL}$ HCT (hematocrit) $=20.9 \%$, WBC (white blood cells) $=5.8210 * 9 / \mathrm{L}$, PLT (platelets) $=263 \quad 10^{*} 9 / \mathrm{L}, \quad$ INR $=16.6$. Myocardial cytolysis and hepatocytolysis enzymes were initially within normal limits, subsequently $\mathrm{TGO}=1459 \mathrm{U} / \mathrm{L}, \mathrm{TGP}=669$ $\mathrm{U} / \mathrm{L}$, Creatinine clearance $=5 \mathrm{ml} / \mathrm{min} / 1.73 \mathrm{~m} 2$ CKD-EPI.

The chest radiography showed medium left pleurisy, cardiomegaly and absence of pulmonary congestion.

The echocardiography shows a normal left ventricular cavity with moderate systolic dysfunction because of the akinesia of the apical $1 / 2$ of the septum, anterolateral wall, anterior wall and apex, associated with moderate mitral regurgitation. The pericardial effusion was circumferentially distributed $(25.3$ $\mathrm{mm}$ anterior to the right chambers, $11 \mathrm{~mm}$ posterior to the left ventricle), and it determined compression of the right atrium. (Figures 1 and 2)

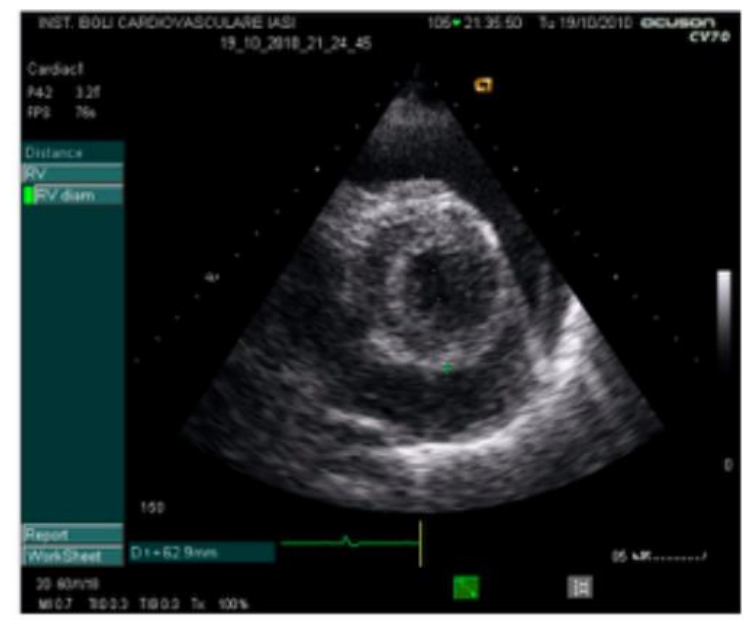

Fig. 1. Echocardiography apical 4 chambers and parasternal short axis sections demonstrate circumferential pericardial effusion and compression of the right atrium 

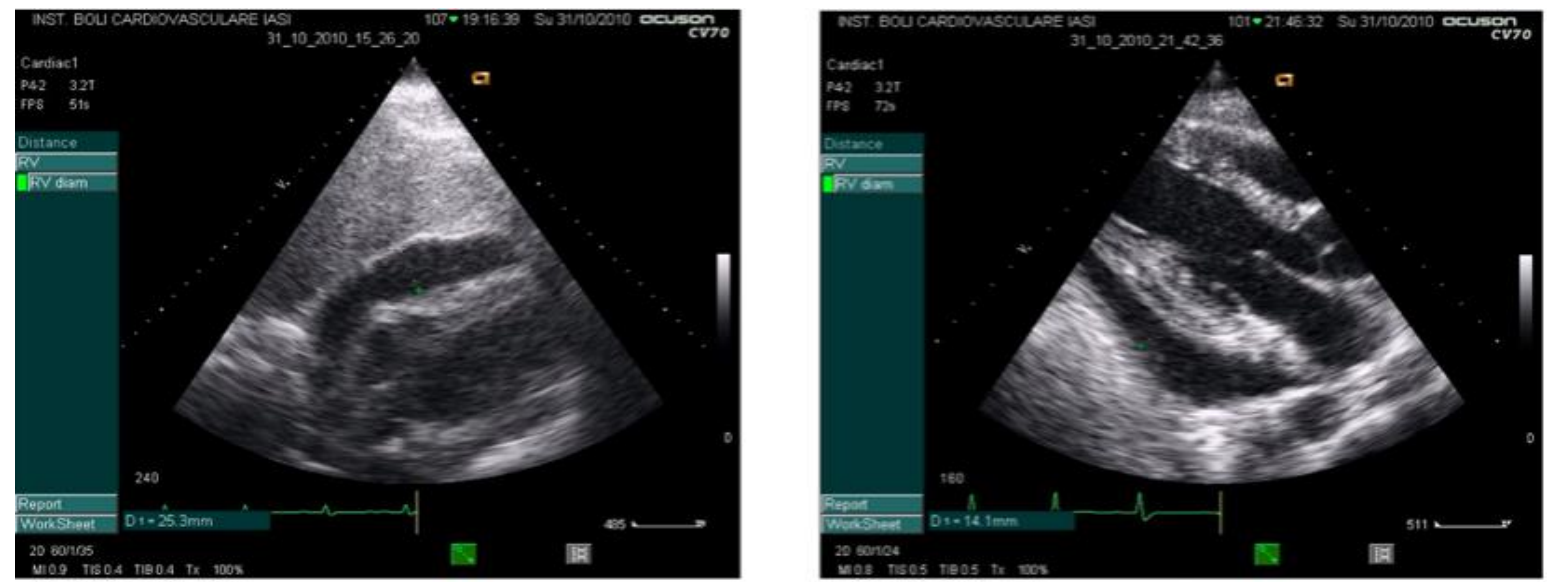

Fig. 2. Echocardiography subcostal and parasternal long axis sections demonstrate pericardial effusion anterior to the right ventricle and posterior to the left ventricle

Diagnostic and evacuation pericardiocentesis was performed and $600 \mathrm{ml}$ of hemorrhagic fluid was evacuated. Postpuncture, the effusion decreased by $10 \mathrm{~mm}$ and the patient's condition enhanced, so the vasopressor therapy was stopped. The pericardial fluid was hemorrhagic with abundant blood mass and cells of venous origin, isolated and agglutinated platelet, $\mathrm{Ht}=$ $30 \%$; proteins $=57 \mathrm{~g} / \mathrm{L}$, glucose $=49 \mathrm{mg} / \mathrm{dL}$, amylase $=37 \mathrm{U} / \mathrm{L}, \mathrm{LDH}=2374 \mathrm{U} / \mathrm{L}, \mathrm{ADA}$ (adenozin deaminaza) $=38 \mathrm{U} / \mathrm{L}$.

Diagnostic and evacuation thoracocentesis was also performed, $900 \mathrm{ml}$ of hemorrhagic fluid was evacuated (proteins $38 \mathrm{~g} / \mathrm{L}$, glucose $=120 \mathrm{mg} / \mathrm{dL}$, amylase $-37 \mathrm{U} / \mathrm{L}$, $\mathrm{LDH}=486 \mathrm{U} / \mathrm{L}, \mathrm{ADA}=25 \mathrm{U} / \mathrm{L}$, cellularity 3080 elements $/ \mathrm{mm} 3$, tumor cells were absent).

The patient's evolution was favorable giving the positive inotropic therapy, oxygen therapy, hemodialysis (HD) with ideal weight loss, blood transfusions, pericardium and pleurocentesis, which led to hemodynamic stabilization, anemic syndrome correction, and remission of pleural-pericardial fluid and hepatocytolysis

\section{Discussions}

The management of ESRD associated with a rhythm disorder that requires chronic oral anticoagulation (OAC) is a challenge for the physician. Hemorrhagic pericarditis is a rare complication that endangers the patient's life.

Hemodialysis is associated with frequent cardiovascular modifications in patients with ESRD. In was shown that during HD, rapid electrolytic changes can occur. [5]

Highly fluctuating levels of potassium, magnesium, ionized calcium and other divalent ions are the most frequent electrolyte abnormalities that can occur in maintenance HD patients. Patients on HD also have fluctuations in bicarbonate, sodium levels and volume status, due to the dialysis procedure that is of intermittent nature. Conventional dialysis leads to a sudden decrease in plasma potassium concentration. That has an effect on the extracellular and intracellular potassium concentration gradient, hypokalemia favoring rhythm disturbance through transmembrane potential and repolarization of the cardiac cells. The most common rhythm disturbance in hemodialyzed patients is AF due to the decrease of the potassium happening in the first hour of HD. [6]

Chronic AF can be found among approximately $14 \%$ of $\mathrm{HD}$ patients, and it may become more prevalent as the dialysis patients continues to age [7]; In the Framingham cohort, during an observation period of 20 years, AF had an incidence of $0.2 \%$ per year.

AF can occur also as a sign of pericarditis, but the rhythm abnormality is almost always caused by constrictive pericarditis.

In our case, we have the two favoring factors for AF: the electrolyte abnormality 
(hypokalemia $\mathrm{K}=3.2 \mathrm{mEql} / \mathrm{L}$ ) caused by the $\mathrm{HD}$ and the pericarditis.

Patients with AF and ESRD are at a higher risk of systemic thromboembolism or stroke compared to those without renal disease. [8] Unfortunately, it is not known if patients undergoing hemodialysis have a clear benefit or are at risk when taking OAC, because they have substantially increased risks of bleeding events, such as hemorrhagic stroke, or, on the other hand, ischemic stroke. [9]

Anemia also plays a pathogenetic role in bleeding risk in patients with ESRD, as its correction leads to improved platelet function in this patient population. [10] It has been shown that the hemodialysis procedure may also activate tPA directly, but it is not known if the activation predisposes to a higher risk of bleeding. [11]

There is no evidence to support the efficacy and safety of OAC's in patients with AF and severe renal disease, because this type of patients was excluded from pivotal clinical trials regarding this matter. [12] Because of the lack of evidence regarding the usage of OAC's in patients with ESRD (GFR $<15 \mathrm{ml} / \mathrm{min}$ ), further research was called by the European guidelines. However, the American guidelines recommended warfarin treatment for this group of patients. [13]

Giving this matter, many studies tried to clarify the correct use of OAC's in patients with $A F$ and ESRD. Hared et al. made a systematic review and meta-analysis concerning the safety and efficacy of VKA's in preventing ischemic stroke for patients associating $\mathrm{AF}$ with ESRD and receiving dialysis. They got to the conclusion that Warfarin was not associated with a clear benefit or harm and suggested further analysis. [14] In Journal of the American College of Cardiology, Bonde et al. found that, no matter the level of renal dysfunction, anticoagulation in patients with $\mathrm{CHA}_{2} \mathrm{DS}_{2}$-VASc score $>2$ was associated with better outcomes. [12]

However, in patients on renal replacement therapy, the all-cause mortality was only a bit reduced. This suggests that was not necessarily an effect of the therapeutic prevention of ischemic stroke, but maybe a confounding of the indication. The results of this study show that, regardless of the
$\mathrm{CHA}_{2} \mathrm{DS}_{2}-\mathrm{VASc}$ score, the risk for stroke or pulmonary embolism is doubled by the presence of CKD. [15] To conclude, the greater the risk of ischemic stroke associated with $\mathrm{AF}$ and background renal disease, the greater the benefit of anticoagulation. [16]

Recently, Hussain S et al. showed, in a meta-analysis and cohort study, that, among patients with $\mathrm{AF}$ and mild-to-moderate renal dysfunction, subgroup analyses from trials and observational studies appear to support the net benefit of VKAs over no OAC treatment. [17]

In this case report, the patient with intradialitic paroxysmal $\mathrm{AF}$ has a $\mathrm{CHA}_{2} \mathrm{DS}_{2}{ }^{-}$ VASc score of 3 and a HAS-BLED score of 5. Although the hemorrhagic risk was higher than the embolic risk, OAC with Acenocumarol was initiated in the regional hospital.

This approach raises two questions. If the paroxysmal AF was a intradialytic event due to hydro-electrolytic changes, does the patient need OAC therapy? And if we don't consider it a singular, intradialytic event, and start OAC isn't the anti-inflammatory treatment going to increase the bleeding risk of a dialyzed patient?

ESRD patients have an increased risk of hemorrhage caused by platelet dysfunction, GPIIb/IIla glycoproteins dysfunction, early degranulation with release of ADP and serotonin, which leads to early platelet adhesion and aggregation. [18] Some uremic toxins such as guanidinosuccinic acid and methyl guanidin may contribute to platelet dysfunction by stimulating the release of nitric oxide.[19]

In our case, this led to hemorrhagic pleural-pericarditis, which we interpreted as having multiple etiologies (hyper-hydration, overdose of Acenocumarol and association between OAC and anti-inflammatory therapy).

Therefore, the patient the patient underwent hemodialysis sessions (4 hours and 15 minutes, flow rate $350 \mathrm{ml} / \mathrm{h}$ ) with additional weight loss and remittance of pericardial and pleural fluid. We also proceeded by stopping the OAC and antiinflammatory treatment.

The 2015 ESC guideline for diagnosis and management of pericardial disease states that patients with ESRD are more likely to have 
chronic pericardial effusion due to volume overload. However, not all pericardial effusion is a result of inflammation, and the volume of pericardial fluid is higher in stable hemodialysis patients than in the control group. The most likely cause of uremic pericarditis is the retention of toxic metabolites. Because pericardial effusion is often bloody in uremic patients, anticoagulation should be considered with great care or avoided in patients starting dialysis. [20]

Initiation of anticoagulant treatment resulted in overdose of Acenocumarol - initial INR 16.6 and bleeding complications. The anticoagulant treatment was stopped, and after the evacuation the evolution was favorable. Holter ECG monitoring during the hospital stay did not found AF.

The use of heparin and anticoagulant therapies is often perceived as a possible risk factor for the development of a hemorrhagic pericardial effusion that can lead to cardiac tamponade. A multivariable analysis of nearly 500 consecutive cases of acute pericarditis did not demonstrate this. Similarly, in a study of 274 patients with acute pericarditis or myopericarditis, the use of heparin or anticoagulants was not associated with an increased risk of cardiac tamponade. On the other hand, within iatrogenic effusive pericarditis, complete anticoagulation may be a risk factor for tamponade and other complications. [20]

Other causes were discussed, but subsequently excluded because we had no arguments:

Tuberculosis. $1250 \mathrm{ml}$ hemorrhagic pleural fluid with slightly increased ADA in the pericardial and pleural fluid was evacuated, but the Quantiferon test was negative. Chest $x$-ray shows no evolutionary lung injury. The patient underwent a pneumological examination, which excluded the diagnosis of pulmonary TB. In dynamics, the pleural fluid was no longer revealed by echocardiography.

Tuberculous pericarditis is responsible for less than $4 \%$ of pericardial diseases in developed countries. In contrast, TB is the etiology of a major pericardial effusion in more than $90 \%$ of those infected with HIV and in 50 $70 \%$ of individuals who are not infected with
HIV, but live in developing countries, where TB is endemic. The disease can occur at any age, and men are more commonly affected than women. The most common clinical form is the one with chronic cardiac compression, thus mimicking congestive heart failure. Clinical manifestations are pericardial effusion, effusive-constrictive pericarditis and constrictive pericarditis. [21]

Neoplasia. No tumor cells were found in the cytological examination of the pleural and pericardial fluid. The patient shows no other signs of neoplastic impregnation, and the explorations performed did not identify a neoplasm.

Neoplasia is the most common cause of hemorrhagic pleuro-pericarditis. Primary pericardial tumors, benign (lipids and fibroids) and malignant (mesotheliomas, angiosarcomas, fibrosarcomas) are very rare. Mesothelioma, the most common malignant pleural tumor, is almost always incurable. The most common secondary malignancies are lung cancer, breast cancer, malignant melanoma, lymphomas and leukemias. [22]

\section{Conclusions}

To conclude, do we treat a patient with ESRD that suffers from paroxysmal AF? And if we do, is a complication such as pleuralpericarditis worth taken? The untreated patient with CKD is generally more prone to thrombosis; however, when treated with antiplatelet agents, heparin, warfarin, and novel anticoagulants, the patient is more prone to bleeding compering with one that has normal renal function.

In this case the decision was made by approaching the risk-benefit ratio of both the hemorrhagic and embolic status, treating the patient according to what was favorable for him at the time. OAC is not recommended for paroxysmal AF caused by hypokalemia when the bleeding risk is high. Also, the evaluation of the hydration status of the HD patient is very important.

\section{Consent}

Written informed consent was obtained from the patient for publication of this case report and accompanying images. A copy of the written 
consent is available for review by the Editor-in-Chief of this journal.

\section{Competing interests}

The author(s) declare that they have no competing interests.

\section{References}

1. Hoit BD. Anatomy and Physiology of the Pericardium. Cardiol Clin 2017; 35(4):481-490.

2. Maisch B. Management of pericarditis and pericardial effusion, constrictive and effusiveconstrictive pericarditis. Herz 2018; 43(7):663678.

3. LeWinter MM. Clinical practice. Acute pericarditis. N Engl J Med 2014; 371(25):24102416.

4. Imazio M, Gaita F. Diagnosis and treatment of pericarditis. Heart 2015; 101(14):1159-1168.

5. Genovesi S, Pogliani D, Faini A, Valsecchi M, Riva A. Prevalence of atrial fibrillation and associated factors in a population of long-term hemodialysis patients. Am J Kidney Dis 2005; 46(5):897-902

6. Voroneanu L, Covic A, Arrhythmias in hemodialysis patients. J Nephrol 2009; 22 (6):716- 725.

7. Vázquez E, Sánchez-Perales C, Borrego F, et al. Influence of atrial fibrillation on the morbidomortality of patients on hemodialysis. Am Heart J 2000; 140:886-890.

8. Goto S, Angchaisuksiri P, Bassand J, et al. Management and 1-year outcomes of patients with newly diagnosed atrial fibrillation and chronic kidney disease: results from the prospective GARFIELD-AF registry. $J A m$ Heart Assoc 2019; 8(3): e010510. doi: 10.1161/JAHA.118.010510.

9. Winkelmayer WC, Patrick AR, Liu J, Brookhart A, Setoguchi S. The increasing prevalence of atrial fibrillation among hemodialysis patients. $J$ Am Soc Nephrol 2011; 22(2):349-357.

10. Hedges SJ, Dehoney SB, Hooper JS, Amanzadeh J, Busti AJ. Evidence-based treatment recommendations for uremic bleeding. Nat Clin Pract Nephrol 2007; 3(3):138-153.

11. Jalal D, Chonchol M, Targher G. Disorders of hemostasis associated with chronic kidney disease. Semin Thromb Hemost 2010; 36(1):34-40.

12. Bonde $A N$, Lip $G Y H$, Kamper $A L$, et al. Net clinical benefit of antithrombotic therapy in patients with atrial fibrillation and chronic kidney disease: a nationwide observational cohort study. J Am Coll Cardiol 2014; 64(23):2471-2482.
13. January CT, Wann LS, Alpert JS, et al. AHA/ACC/HRS guideline for the management of patients with atrial fibrillation: a report of the American College of Cardiology/American Heart Association Task Force on Practice Guidelines and the Heart Rhythm Society. J Am Coll Cardiol 2014; 64(21):e1-76.

14. Harel Z, Chertow GM, Shah PS, et al. Warfarin and the risk of stroke and bleeding in patients with atrial fibrillation receiving dialysis: a systematic review and meta-analysis. Can $J$ Cardiol 2017; 33:737-746.

15. Adams MJ, Irish AB, Watts GF, Oostryck R, Dogra GK. Hypercoagulability in chronic kidney dis- ease is associated with coagulation activation but not endothelial function. Thromb Res 2008; 123:374-380.

16. Ball $\mathrm{T}$, Wheelan $\mathrm{K}$, McCullough P. Chronic anticoagulation in chronic kidney disease. J Am Coll Cardiol 2014; 64(23): 2483-2485.

17. Hussain S, Siddiqui AN, Baxi H, Habib A, Hussain MS, Najmi AK. Warfarin use increases bleeding risk in hemodialysis patients with atrial fibrillation: a meta-analysis of cohort studies. $J$ Gastroenterol Hepatol 2019; 34(6):975-984.

18. Małyszko J, Małyszko JS, Myliwiec M. Endothelial cell injury markers in chronic renal failure on conservative treatment and continuous ambulatory peritoneal dialysis. Kidney Blood Press Res 2004; 27(2):71-77.

19. Huang $\mathrm{Y}$, Noble NA. PAI-1 as a target in kidney disease. Curr Drug Targets 2007; 8(9):1007-1015.

20. Adler $Y$, Charron $P$, Imazio M, et al. 2015 ESC Guidelines for the diagnosis and management of pericardial diseases: The Task Force for the Diagnosis and Management of Pericardial Diseases of the European Society of Cardiology (ESC), The European Association for Cardio-Thoracic Surgery (EACTS). Eur Heart J 2015; 36(42):2921-2964.

21. Ntsekhe M, Mayosi BM. Tuberculous pericarditis with and without HIV. Heart Fail Rev 2013; 18(3):367-373.

22. Restrepo CS, Vargas D, Ocazionez D, Martínez-Jiménez S, Betancourt Cuellar SL, Gutierrez FR, Primary pericardial tumors. Radiographics 2013; 33(6):1613-1630. 